\title{
Reconstruction of Tomographic Images through Machine Learning Techniques ${ }^{\dagger}$
}

\author{
Xosé Fernández-Fuentes ${ }^{1, *, \neq}$, David Mera ${ }^{1, \ddagger}$ and Andrés Gómez ${ }^{2, \ddagger}$ \\ 1 Centro Singular de Investigación en Tecnoloxías da Información (CiTIUS), Universidade de Santiago de \\ Compostela, Rúa de Jenaro de la Fuente Domínguez, 15782 Santiago de Compostela, Spain; \\ david.mera@usc.es \\ 2 Galicia Supercomputing Center (CESGA), 15705 Santiago de Compostela, Spain; agomez@cesga.es \\ * Correspondence: xosefernandez.fuentes@usc.es; Tel.: +34-881-816-390 \\ + Presented at the XoveTIC Congress, A Coruña, Spain, 27-28 September 2018. \\ $\ddagger$ These authors contributed equally to this work.
}

Published: 17 September 2018

check for updates

\begin{abstract}
Some problems in the field of health or industry require to obtain information from the inside of a body without using invasive methods. Some techniques are able to get qualitative images. However, these images are not enough to solve some problems that require an accurate knowledge. Normally, the tomography processes are used to explore inside of a body. In this particular case, we are using the method called Electrical Impedance Tomography (EIT). The basic operation of this method is as follows: (1) The electrical potential difference is measured in the electrodes placed around the body. This part is known as forward model. (2) Get information from the inside of the body using the measured voltages. This problem is known as inverse problem. There are several approximations to solve this inverse problem. However, these solutions are focused on obtaining qualitative images. In this paper, we show the main challenges of how to obtain quantitative knowledge when Machine Learning techniques are used to solve this inverse problem.
\end{abstract}

Keywords: Electrical Impedance Tomography; Machine Learning; Artificial Neural Networks; inverse problems

\section{Introduction}

Certain medical and industrial problems need to get information from the inside of a body without damaging it. There are techniques able to get qualitative images about the distribution of some physical characteristic of a particular body. However, these images are not useful to solve problems that require a quantitative knowledge of a concrete physical feature. One of the ways to get this knowledge is using tomography processes, which explore the inside of a body in a non-invasive way. In this paper, we have focused our efforts on the tomography process called Electrical Impedance Tomography (EIT). We have chosen EIT because it can be useful for both medicine and industry due to the fact it is easy to deploy.

Signals obtained through a tomography process are used to solve an ill-posed [1,2] nonlinear inverse problem with the purpose of obtaining the distribution of a physical characteristic. To deal with this mathematical problem exist different approaches. On the one hand, some techniques apply iterative algorithms [3,4]. They are quite accurate but they demand a lot of time and large computing capacity. On the other hand, there are some algorithms that assume some linearity in the response of the body. These algorithms are fast but inaccurate $[5,6]$.

In this paper, we show what are the main challenges to solve this inverse problem in an accurate and fast way thanks to Machine Learning (ML) techniques. Some previous work [7-9] explore the 
possibility of using ML to solve the inverse problem. However, their focus is on reconstructing the images from a qualitative point of view instead of quantitative.

\section{Challenges}

The first problem is to get an appropriate dataset. Because there is not a dataset large enough to train the models, it is necessary to generate it. This new dataset must contain simulations of bodies with distributions and volumes of different physical properties. In addition, it is necessary to take into account the position and dimensions of the electrodes used. Once this set has been generated, it is necessary to simulate the tomographic processes to each body. In the case of EIT, the software EIDORS [10] is appropriate to perform this simulation. After the simulation is done, noise should be added to make the signals more similar to those obtained in a real environment. This implies studying and defining an appropriate function that allows the signals to be distorted in an appropriate way.

The second challenge is to train different algorithms of Machine Learning, with the objective of making a comparison that allows identifying the algorithms that best adapt to this type of highly non-linear problem. It must be taken into account that the final algorithm must be robust to the noise of the sensors, the body shape and the position of the sensors. Furthermore, to be able to train so many different models, it is necessary to have available a large computing capacity and a lot of storage. At the same time, this implies to carry out an efficient management of the computational resources.

The third challenge (very related to the previous one) is to develop adequate metrics for the training of the algorithms. Traditional metrics do not seem appropriate due to the atypical unbalance between inputs and outputs (many more outputs than inputs).

The fourth problem is the validation of the final algorithm. This implies testing the model with a real test set that has been obtained through tomographic tests.

\section{Results}

We have done some initial tests using Artificial Neural Networks. To perform these first tests, we have made various simplifications. For example, we have maintained the same body shape and we have not introduced noise in the measurements of the sensors. Having this in mind, the results obtained are very interesting and promising taking into account the complex nature of the problem.

Acknowledgments: This work has received financial support from the Predoctoral scholarship program of the Xunta de Galicia (ED481A-2018/277), the Xunta de Galicia under Research Network R2016/045, the Consellería de Cultura, Educación e Ordenación Universitaria (accreditation 2016-2019, ED431G/08) and the European Regional Development Fund (ERDF). Computational resources were provided by the Galicia Supercomputing Center (CESGA).

Conflicts of Interest: The authors declare no conflict of interest. The founding sponsors had no role in the design of the study; in the collection, analyses, or interpretation of data; in the writing of the manuscript, and in the decision to publish the results.

\section{References}

1. Michalikova, M.; Abed, R.; Prauzek, M.; Koziorek, J. Image Reconstruction in Electrical Impedance Tomography Using Neural Network. In Proceedings of the Biomedical Engineering Conference (CIBEC), Giza, Egypt, 11-13 December 2014; pp. 39-42.

2. Wang, P.; Li, H.L.; Xie, L.L.; Sun, Y.C. The implementation of FEM and RBF neural network in EIT. In Proceedings of the 2nd International Conference on Intelligent Networks and Intelligent Systems (ICINIS 2009 ), Tianjin, China, 1-3 November 2008; Volume 3, pp. 66-69.

3. Wang, C.; Lang, L.; Wang, H.-X. RBF neural network image reconstruction for electrical impedance tomography. In Proceedings of the 2004 International Conference on Machine Learning and Cybernetics, Shanghai, China, 26-29 August 2004; Volume 4, pp. 2549-2552.

4. Adler, A.; Guardo, R. A Neural Network Image Reconstruction Technique for Electrical Impedance Tomography. IEEE Trans. Med. Imaging 1994, 13, 594-600. 
5. Wu, K.; Yang, J.; Dong, X.; Fu, F.; Tao, F.; Liu, S. Comparative study of reconstruction algorithms for electrical impedance tomography. IEEE Trans. Biomed. Eng. 2012, 51077127, 2296-2299.

6. Guardo, R.; Boulay, C.; Murray, B.; Bertrand, M. An experimental study in electrical impedance tomography using backprojection reconstruction. IEEE Trans. Biomed. Eng. 1991, 38, 617-627.

7. Liu, X.; Wang, X.; Hu, H.; Li, L.; Yang, X. An extreme learning machine combined with Landweber iteration algorithm for the inverse problem of electrical capacitance tomography. Flow Meas. Instrum. 2015, 45, 348-356.

8. Martin, S.; Choi, C.T. Nonlinear Electrical Impedance Tomography Reconstruction Using Artificial Neural Networks and Particle Swarm Optimization. IEEE Trans. Magn. 2016, 52, 1-4.

9. Martin, S.; Choi, C.T.M. A Post-Processing Method for Three-Dimensional Electrical Impedance Tomography. Sci. Rep. 2017, 7, 7212.

10. Adler, A.; Lionheart, W.R. Uses and abuses of EIDORS: An extensible software base for EIT. Physiol. Meas. 2006, 27, S25.

(C) 2018 by the authors. Licensee MDPI, Basel, Switzerland. This article is an open access article distributed under the terms and conditions of the Creative Commons Attribution (CC BY) license (http:/ / creativecommons.org/licenses/by/4.0/). 